\title{
Semi-automated myocardial segmentation of bright blood multi-gradient echo images improves reproducibility of myocardial contours and $\mathrm{T} 2 *$ determination
}

\author{
Pandji Triadyaksa $^{1,3}$ (1) Niek H. J. Prakken ${ }^{1,2} \cdot$ Jelle Overbosch $^{2} \cdot$ Robin B. Peters $^{2} \cdot$ \\ J. Martijn van Swieten ${ }^{2} \cdot{\text { Matthijs } \text { Oudkerk }^{1} \cdot \text { Paul E. Sijens }}^{1,2}$
}

Received: 14 June 2016 / Revised: 23 November 2016 / Accepted: 24 November 2016 / Published online: 16 December 2016

(C) The Author(s) 2016. This article is published with open access at Springerlink.com

\begin{abstract}
Objectives Early detection of iron loading is affected by the reproducibility of myocardial contour assessment. A novel semi-automatic myocardial segmentation method is presented on contrast-optimized composite images and compared to the results of manual drawing.

Materials and methods Fifty-one short-axis slices at basal, mid-ventricular and apical locations from 17 patients were acquired by bright blood multi-gradient echo MRI. Four observers produced semi-automatic and manual myocardial contours on contrast-optimized composite images. The semi-automatic segmentation method relies on vector field convolution active contours to generate the endocardial contour. After creating radial pixel clusters on the myocardial wall, a combination of pixel-wise coefficient of variance $(\mathrm{CoV})$ assessment and $k$-means clustering establishes the epicardial contour for each segment.

Results Compared to manual drawing, semi-automatic myocardial segmentation lowers the variability of $\mathrm{T} 2 *$ quantification within and between observers ( $\mathrm{CoV}$ of 12.05 vs. $13.86 \%$ and 14.43 vs. $16.01 \%$ ) by improving contour reproducibility $(P<0.001)$. In the presence of iron loading, semi-automatic segmentation also lowers the $\mathrm{T} 2 *$
\end{abstract}

Pandji Triadyaksa

p.triadyaksa@umcg.nl

1 Center for Medical Imaging-North East Netherlands, University of Groningen, University Medical Center Groningen, EB45, 30001, 9700 RB Groningen, The Netherlands

2 Department of Radiology, University of Groningen, University Medical Center Groningen, EB45, 30001, 9700 RB Groningen, The Netherlands

3 Department of Physics, Diponegoro University, Sudharto Street, Semarang 50275, Indonesia variability within and between observers ( $\mathrm{CoV}$ of 13.14 vs. $15.19 \%$ and 15.91 vs. $17.28 \%$ ).

Conclusion Application of semi-automatic myocardial segmentation on contrast-optimized composite images improves the reproducibility of $\mathrm{T} 2 *$ quantification.

Keywords Magnetic resonance imaging - Iron loading · Bright blood myocardial T2* $\cdot k$-Means clustering $\cdot$ Vector field convolution active contour

\section{Introduction}

Cardiovascular magnetic resonance imaging (MRI) techniques are used for non-invasive assessment of patients with iron loading in the heart, i.e. thalassemia, hemochromatosis, cardiomyopathy and sickle cell disease [1-5]. The assessment includes multi-gradient echo (MGE) imaging to quantify myocardial $\mathrm{T} 2 *$ in bright blood as well as black blood modes [3-7]. Recent assessments of iron loading feature the mid-ventricular septum as well as other areas of the myocardium $[1,8,9]$.

Myocardial iron loading identification at an early stage is important to prevent cardiac complication [8] leading to heart failure [10]. Assessment of the entire myocardium rather than just the septum enables the prediction of iron loading at an early stage $[1,8]$, also considered relevant for the characterization of pathology in post-mortem studies [11-14]. In the global assessment, susceptibility artifacts are frequent in posterior lateral and anterior regions, but may be corrected for to ensure reliable myocardial T2* values [15-18].

In the assessment of myocardial iron deposition, manual myocardial contour drawing is time-consuming and subject to intraobserver and interobserver variability. Several 
segmentation methods have been developed to produce automatic left ventricular (LV) myocardial contours [19], but their accuracy is influenced by tissue contrast quality that depends on intrinsic tissue parameters, scanning hardware, sequence type, and imaging parameters [20]. Imagebased (i.e. $k$-means clustering) and contour-based (i.e. active contours) approaches are widely used in LV myocardial segmentation but their application is sensitive to image intensity and contrast variability or requires a large training dataset to account for anatomical and imaging variations [20]. Using prior knowledge of LV morphology, the application of these two approaches has been effective to automatically determine the LV myocardial contours for various MR parameters and settings [19].

Commercial software packages do feature automatic methods in their delineation option but, to our knowledge, are focused on myocardial MR cine imaging [21-25] and are not specifically designed for myocardial $\mathrm{T} 2 *$ assessment. Recently, an automatic method was presented for segmenting the mid-ventricular region for myocardial T2* measurement using black blood $[17,26]$ and bright blood [27] MGE series. Even though the black blood mode is more favorable for iron loading quantification [7], the bright blood mode is still widely used as a standard sequence for large populations $[1,8]$ and follow-up studies $[28,29]$. In bright blood mode, myocardial segmentation remains a challenge due to the poor contrast between the myocardium and its surroundings on the original MGE images [19]. The use of a single image with the shortest echo time (TE) as a template for morphological operation still produces high observer variability as reported elsewhere [27]. The combination of eight MGE images acquired with different TEs to increase the detectability of the myocardial boundary information was proposed, but without documentation of the obtained contrast improvement [27]. As an alternative, a composite image offering better contrast [30] can serve as a template image. The use of a composite image (Fig. 1i) as a combination of three images representing up to three TEs from the MGE series (Fig. 1a-h) has recently been shown to reduce observer variability in drawing LV epicardial and endocardial contours [30] compared with the common practice of using a single short-axis image corresponding to a single TE $[3,7$, $8,27]$. This procedure, while improving the reproducibility of myocardial contours, is still observer-dependent. This study aims to resolve the difficulties of myocardial $\mathrm{T} 2$ * assessment in a bright blood MGE series by improving the generation of contrast-optimized composite images and use it as a template for image-based and contour-based semiautomatic segmentation, combined with prior knowledge of LV morphology, to advance the reproducibility of LV myocardial contour and $\mathrm{T} 2 *$ quantification.

\section{Materials and methods}

\section{Patients}

This retrospective study involved post-processing of clinical data acquired between February 2009 and September 2015, with approval from the hospital review board that waived informed consent. In this period, 22 patients were examined by a clinical routine cardiac MRI protocol including MGE with three short-axis slices at apical, mid-ventricular, and basal locations. Five patients were excluded due to motion blurring. The remaining 17 patients consisted of 6 cases of thalassemia, 6 with hemochromatosis, 3 with suspected cardiomyopathy, 1 with sickle cell disease, and 1 congenital dyserythropoietic anaemia patient.

\section{Multi-gradient echo magnetic resonance imaging}

Cardiac MRI studies were performed at 1.5 Tesla (T) using one of two Siemens scanners (Siemens Medical
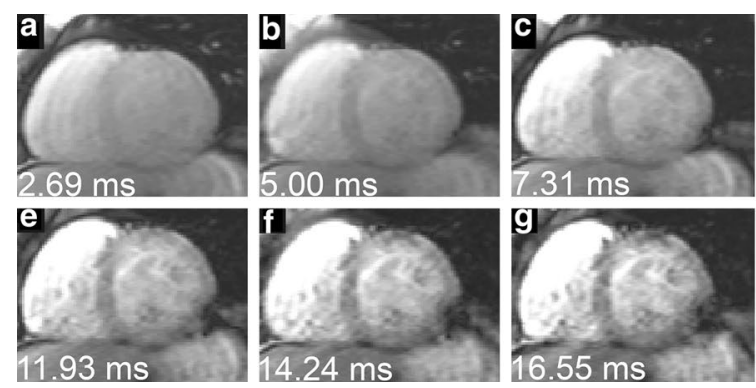

Fig. 1 Eight short-axis images of a bright blood multi-gradient echo series (a-h) and the contrast-optimized composite image (i) generated by combining the image at an echo time (TE) of $2.69 \mathrm{~ms}$, as a representation of optimum contrast between the myocardium and lung, $14.24 \mathrm{~ms}$ for optimum contrast between the myocardium and right ventricle blood pool and $18.86 \mathrm{~ms}$ for optimum contrast
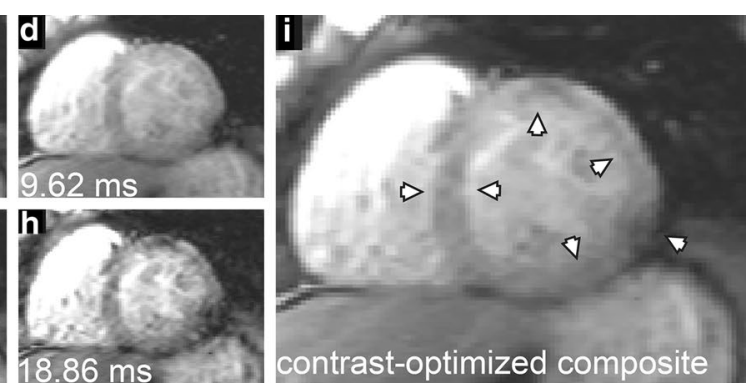

between the myocardium and left ventricle blood pool. Compared to any single TE image, contrast improvements at epicardial and endocardial borders, especially at the free wall myocardium, show on the composite image where at the inferior epicardial border gradient echo susceptibility artifacts are not prominent (contrast improvements highlighted by arrow heads) 
Fig. 2 Steps of contrast-tonoise ratio calculations on multi-gradient echo images by using method $1(\mathbf{a}, \mathbf{b})$ to define regions of interests (ROIs) of left ventricle blood pool (LVBP), right ventricle blood pool (RVBP), and lung. An example of the ROIs generated by method 1 (c) is presented
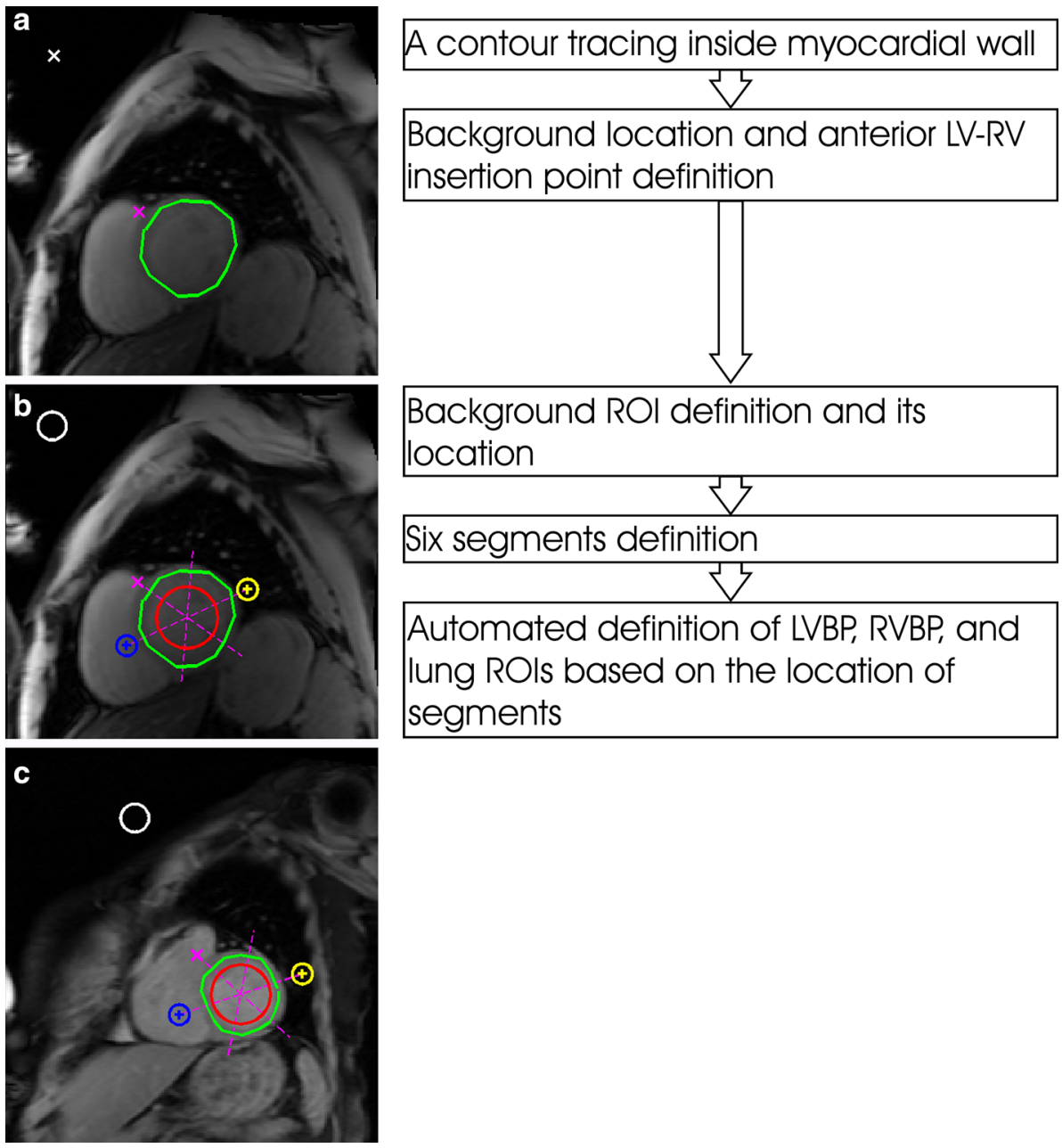

Solution, Erlangen, Germany), between 2009 until 2011 the Avanto in seven patients, and from 2012 until 2015 the Aera in ten patients. A body matrix coil of 6 to 9 elements and a spine matrix coil of 12 to 24 elements were used depending on the chosen field of view (FOV). A single breath-hold bright blood MGE sequence was performed at eight TEs $(2.59-18.20$ at 2.23-ms increments using the Siemens Avanto and 2.69-18.86 at 2.31-ms increments using the Siemens Aera) with a repetition time (TR) of $200 \mathrm{~ms}$ and a flip angle of $20^{\circ}$. On both scanners, a variable FOV of $275-362 \times 400 \mathrm{~mm}^{2}$ was applied according to patient size with a reconstructed voxel size of $1.56 \times 1.56 \times 10 \mathrm{~mm}^{3}, 50 \%$ phase resolution sampling, using 18-24 cardiac cycles per breath-hold with 5 segments in each heart beat and without enabling parallel imaging. Pixel bandwidth was set at $814 \mathrm{~Hz}$ for the Avanto scanner and $815 \mathrm{~Hz}$ for the Aera scanner. From the included 17 patients a total of $51 \mathrm{LV}$ short-axis slices were acquired.

\section{Composite image generation}

In this study, the improvement of contrast-optimized composite images generation was conducted semi-automatically by two methods (Figs. 2, 3) using custom-written software (developed in MATLAB version 7.14, The MathWorks, Natick, MA, USA).

The first method involved tracing a manual contour inside the LV myocardial wall, rather than exactly on the endocardial and epicardial borders, on the shortest TE image from the MGE series to acquire the LV signal intensity along its path, locating a coordinate of air background staying clear of any visible artefact, and locating the coordinate of the anterior LV and right ventricle (RV) insertion point [18]. In Fig. 2a, this is illustrated for a mid-ventricular short axis slice. On the indicated location of air background, an ROI was automatically generated. Based on the insertion location, the LV myocardium was automatically divided into four segments (anterior, septal, inferior, 
Fig. 3 Steps of contrast-tonoise ratio calculations on multi-gradient echo images by using method $2(\mathbf{a}, \mathbf{b})$ to define regions of interests (ROIs) of left ventricle blood pool (LVBP), right ventricle blood pool (RVBP), and lung. An example of the ROIs generated by method 2 (c) is presented

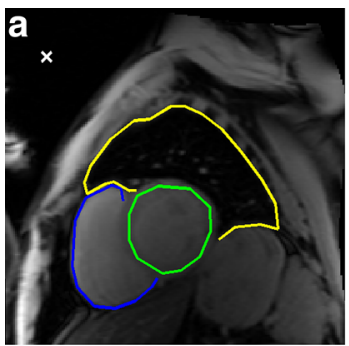

Contour tracing at the borders of RVBP, lung, and inside myocardial wall

Background location definition
Automated definition of LVBP and RVBP ROIs
Automated definition of points in the lung
Automated selection of lung ROI at a point free from
blood vessels

blood vessels

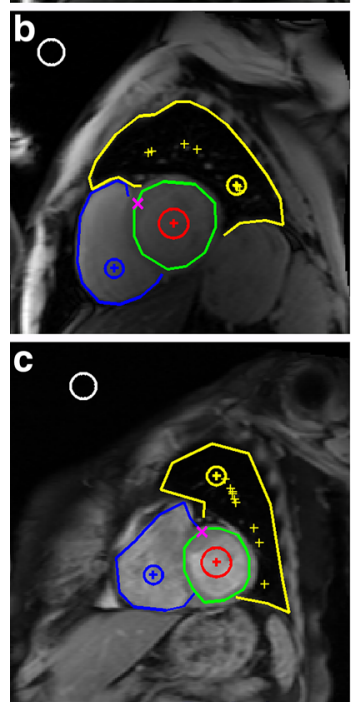

and lateral) at an apical slice, and six segments (anterior, anteroseptal, inferoseptal, inferior, inferolateral, and anterolateral) at basal and mid-ventricular slices. An ROI of LV blood pool (LVBP) was automatically located at the crosssection of the segments inside the blood pool. The right ventricle blood pool (RVBP) ROI was automatically positioned by extrapolating the anteroseptal and inferoseptal segment junction into the nearby RVBP, and the lung ROI was ascertained by extrapolating the inferolateral and anterolateral segment junction into nearby lung (Fig. 2b). An exception is on the apical slice; due to the small area of the RVBP and lung, the ROIs of the RVBP and lung were generated automatically after manually marking their approximate locations. All ROIs and LV myocardial wall contours were automatically propagated through the MGE image series.

The second method required a location of air background while staying clear of imaging artefacts, manual contouring inside the LV myocardial wall, contouring the RVBP, and lung regions on the shortest TE image from the MGE series (Fig. 3a). The anterior LV and RV insertion points were established automatically at the end of the anterior RVBP contour. An ROI at the center of the LVBP was defined by the coordinate of LV myocardial contour. The complete RVBP area was identified combining the septal myocardium and the RVBP contours. The whole lung area was derived from the lateral myocardium and lung contours. The coordinates of RVBP and lung contours defined an ROI at the center of the RVBP and an ROI in the lung. At the lung, the ROI was taken in an area without blood vessels from one of several possible locations (Fig. 3b). The appointed ROIs of LVBP, RVBP, lung, and the LV myocardial wall contour were then automatically propagated through the MGE image series. This method was applied on all three (apical, mid-ventricular and basal) short-axis slices.

After acquiring the signal intensity of the LV myocardial wall, LVBP, RVBP, and lung through the MGE series, contrast-to-noise ratio (CNRs) between the $\mathrm{LV}$ myocardium and its surroundings were defined as follows [30]:

$\mathrm{CNR}=\mathrm{NF} \frac{\left|\mathrm{SI}_{\mathrm{s}}-\mathrm{SI}_{\mathrm{m}}\right|}{\sigma_{\mathrm{b}}}$

where $\mathrm{NF}, \mathrm{SI}_{\mathrm{s}}, \mathrm{SI}_{\mathrm{m}}$, and $\sigma_{\mathrm{b}}$ represent the noise factor [30], the signal intensities of the surroundings of the LV myocardium (LVBP, RVBP, and lung), the signal intensity of LV myocardium, and the standard deviation of air background, respectively. Next, a contrast-optimized composite image was generated from the images of optimum CNR [30]. In the example of Fig. 1, the composite image (Fig. 1i) was thus generated by combining the images of TE $18.86 \mathrm{~ms}$ (Fig. 1h), $14.24 \mathrm{~ms}$ (Fig. 1f), and $2.69 \mathrm{~ms}$ (Fig. 1a) that represents an optimum myocardial CNR relative to its surroundings (LVBP, RVBP, and lung). 
Fig. 4 Semi-automatic myocardial segmentation process
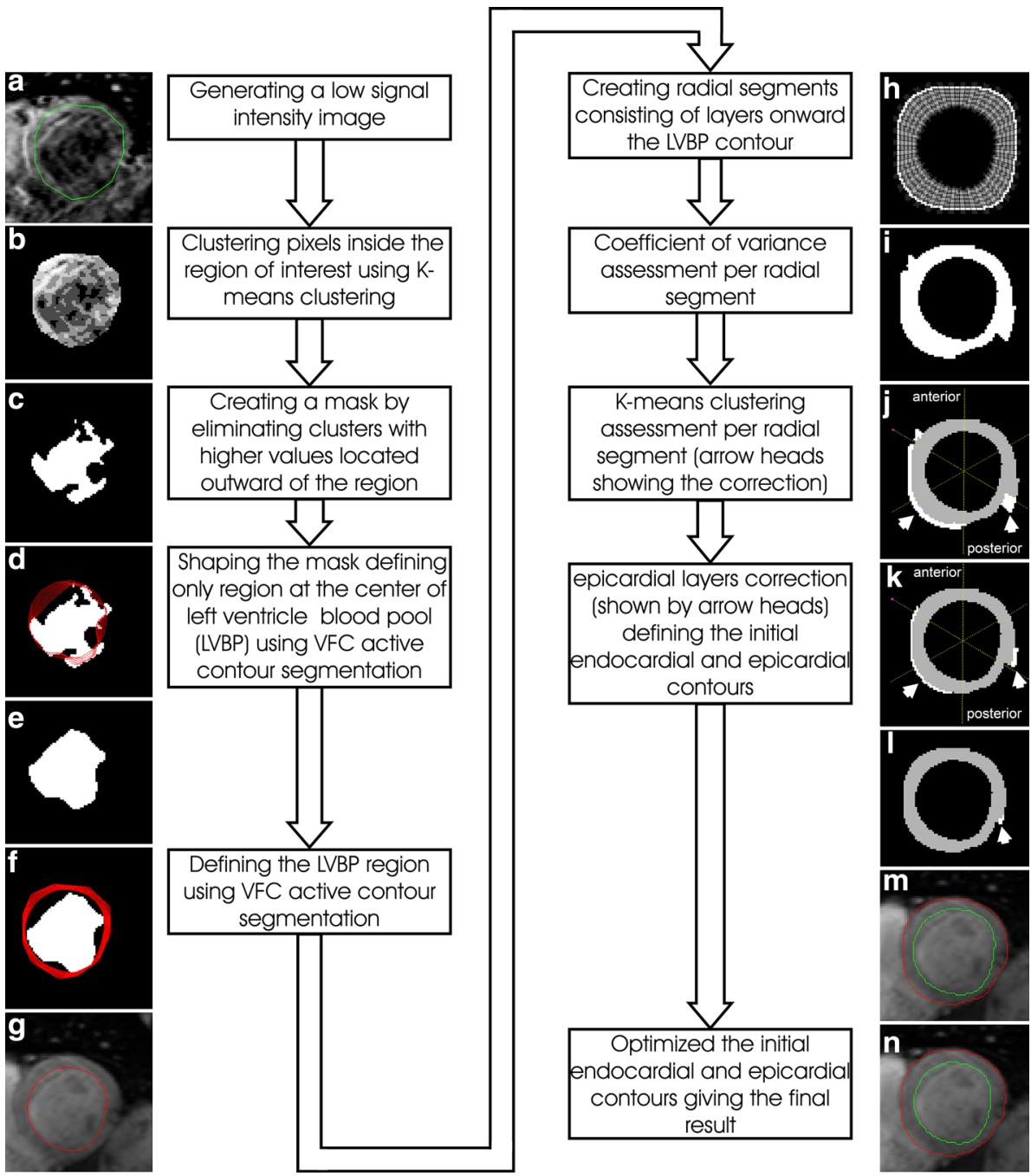

\section{Semi-automatic segmentation}

The generation of the contrast-optimized composite images is a semi-automatic procedure, while the actual segmentation process is fully automated. The processes involve ten steps adding up into two main parts (Fig. 4).

\section{Part I. LVBP region definition}

This part starts by defining the LVBP location on the shortaxis image. In this study featuring a bright blood MGE series, blood signal artefacts, partial volume effects, and the low contrast difference with myocardium make the center definition of LVBP difficult to determine. Therefore, a low-signal-intensity image is proposed (step 1), inspired by the use of blood signal suppression on the black blood image $[7,26]$, to produce signal homogeneity at the LVBP area with adequate contrast between the blood pool and myocardium. Benefiting from the different transverse magnetization decay rate of the LVBP and the myocardium, the image is created by subtracting the shortest TE image with the optimum TE between the myocardium and the LVBP (Fig. 4a). Using the ROI of the myocardium defined earlier (Figs. 2a, 3a), the center of the LVBP is then located by $k$-means clustering (step 2) [31, 32]. Using this clustering, the pixels inside the ROI are grouped based on signal intensity (Fig. 4b) and a mask of the remaining blood pool area at the center is then determined (step 3; Fig. 4c) by removing high cluster values representing myocardial tissue and any artefacts. Afterward, a vector field convolution (VFC) active contour segmentation [33, 34] is applied (step 4; Fig. 4d) to shape the blood pool region (Fig. 4e) which is used as a mask for the subsequent active contour segmentation (step 5; Fig. 4f) to determine the LVBP ROI including papillary muscles and trabeculae (Fig. $4 \mathrm{~g}$ ).

\section{Part II. Myocardial borders definition}

From the LVBP ROI, a mask of one-pixel-thick layers extending beyond any possible myocardial area is created 
by using the signal intensity of the composite image (step 6; Fig. 4h). A maximum normal short-axis LV myocardial thickness of $9.6 \pm 1.6 \mathrm{~mm}$ at basal, $8.5 \pm 1.2 \mathrm{~mm}$ at midventricular, and $6.6 \pm 1.2 \mathrm{~mm}$ at apical level as reported by Kawel et al. [35] is used in this study as expansion limits to create mask layers of $14 \mathrm{~mm}$ at basal and mid-ventricular slices, and $8 \mathrm{~mm}$ at maximum for apical slices. On the first layer adjacent to the LVBP ROI, 72 radial segments on the mid-ventricular and basal slices, and 40 segments on the apical slices are created to ensure that each segment is only filled by up to 2 stacks of pixels measured at the inner layer (Fig. 4h). These amounts of radial segments were chosen to detect the myocardial contour up to pixel level.

Prior knowledge of LV morphology detects signal heterogeneity at outer layers representing the area outside the myocardium. Therefore, coefficients of variance $(\mathrm{CoV})$ in pixel signal intensity, expressed as a standard deviation divided by their mean, are assessed per layer of expansion at each radial segment (step 7). Two adjacent segments at the innermost layer with the smallest $\mathrm{CoV}$ are defined as a starting point for the $\mathrm{CoV}$ assessment. Initial epicardial border expansion stops as soon as the $\mathrm{CoV}$ of the layer's signal intensity exceeds $20 \%$, an empirically optimized threshold. Owing to the high difference of signal intensity between the myocardium and lung, the initial lateral epicardial border was thus located properly as shown in Fig. 4i. The initiation of the epicardial border at anterior, septal and posterior positions, inaccurate due to the low contrast difference at the border, is refined by assessing a $k$-means clustering from the layer expansion of the $\mathrm{CoV}$ assessment (step 8) to create two clusters. After that, the inner cluster is selected as the initial epicardial contour (radial clusters in white colour are excluded from the myocardial region as shown by the arrow head in Fig. 4j).

Further correction is applied (step 9) to eliminate false areas at outer layer(s) of anterior, septal and posterior positions that contain less than $70 \%$ of the maximum number of pixels per layer (a pixel layer in white colour is excluded as shown by left arrow head in Fig. 4k). Meanwhile, the lateral position is corrected similarly with a cut-off value of $40 \%$ (excluding two pixel layers at the right arrow in Fig. 4k). By maximizing the difference in the number of layers per radial segment relative to each neighbour at two (arrow head in Fig. 41), initial endocardial and epicardial contours are established (Fig. $4 \mathrm{~m}$ ).

For a more precise setting of the myocardial contours, two pixel layers inward and outward of the initial epicardial and endocardial contours are added (step 10), creating several combinations of myocardial thickness expansion and compression. The layer expansion or compression serves to test the homogeneity of myocardial area at the borders relative to the whole myocardium. For each combination, pixels in the layers having signal intensities beyond $0.9825 \times \mathrm{Q} 1$ of the interquartile range (IQR) and $0.9825 \times \mathrm{Q} 3$ of the IQR (empirically established thresholds) are counted and identified as outliers. The combination of myocardial layers having the minimum ratio of outlier pixels to total myocardial pixels is then selected as the new myocardial area at the borders. The outlier pixels in the selected layer's combination, indicating the area outside or on the edge of the myocardium, are excluded and optimal endocardial and epicardial contours identified (Fig. $4 \mathrm{n}$ ). In the example of Fig. 4n, optimal contours are acquired by adding one layer at the endocardial border inward to the LVBP and removing outlier pixels at the epicardial border. The optimal myocardial contours as generated by the semi-automatic segmentation are comparable with manual drawing as can be seen in Fig. 5.

\section{T2* quantification}

A monoexponential fitting model with a constant offset was used by the software to quantify pixel-wise myocardial T2* [4]:

$y=K e^{-\mathrm{TE} / \mathrm{T} 2^{*}}+C$

where $y, K, \mathrm{TE}, \mathrm{T} 2 *$, and $C$ represent signal intensity, a fitting constant, echo time, myocardium transverse relaxation time, and a constant of offset correction, respectively. Monoexponential fitting with a constant is known to reduce underestimation of $\mathrm{T} 2 *$ values at higher iron concentrations, both in pixel-wise, and region-based quantifications [4]. Pixel-wise $\mathrm{T} 2 *$ was calculated for four apical, six midventricular and six basal segments, according to the American Heart Association (AHA) 16-segment model [8] using median rather than mean $\mathrm{T} 2 *$ values to reduce the influence of pixel-wise noise and artefact errors [36, 37]. The AHA segments were defined by using the anterior LV and RV reference points of the composite image generation. Global T2* was defined as an average value over 16 segments, and the mid-ventricular septal $\mathrm{T} 2 *$ was the average $\mathrm{T} 2 *$ value of the mid-anterior septum and mid-inferior septum segments.

\section{Reproducibility assessment of myocardial segmentation}

By using the custom-written software as described above, two radiologists (NHJP and JO), one radiology resident (RBP), and one experienced MR technician (JMvS), generated the contrast-optimized composite images by either method. For the intraobserver variability evaluation, both methods were repeated once. Manual drawing of the LV epicardial and endocardial contours on the two sets of composite images from each method was done with an interval of at least 1 week in between. The first author with 6 years 
Fig. 5 An overlay of four myocardial contours assessed by four observers on a contrastoptimized composite image generated by method 2 by manual drawing at apical (a, c), mid-ventricular (e, g) and basal locations (i, k) with its comparison by using semi-automatic segmentation (b and $\mathbf{d}$ at apical, $\mathbf{f}$ and $\mathbf{h}$ at mid-ventricular, and $\mathbf{j}$ and $\mathbf{I}$ at basal locations). Representative contours on slices in the presence $(\mathrm{T} 2 * \leq 20 \mathrm{~ms})$ and absence of iron deposition (T2* $>20 \mathrm{~ms}$ ) in any segment can be seen in $\mathbf{a}, \mathbf{b}, \mathbf{e}, \mathbf{f}, \mathbf{i}, \mathbf{j}$, and $\mathbf{c}, \mathbf{d}, \mathbf{g}$, $\mathbf{h}, \mathbf{k}, \mathbf{l}$, respectively
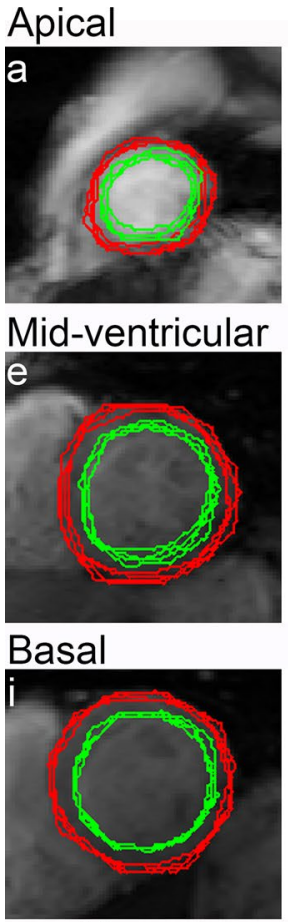

Manual
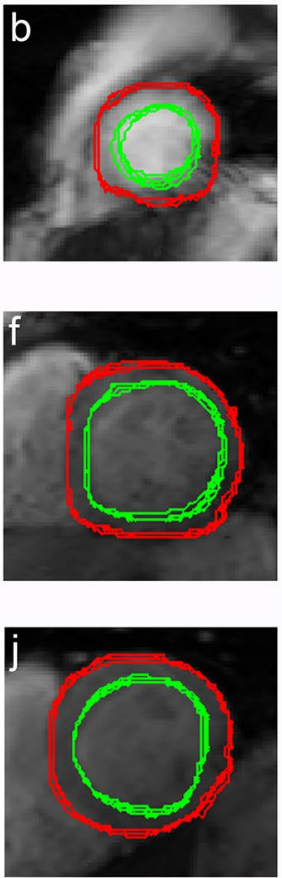

Semi-automatic
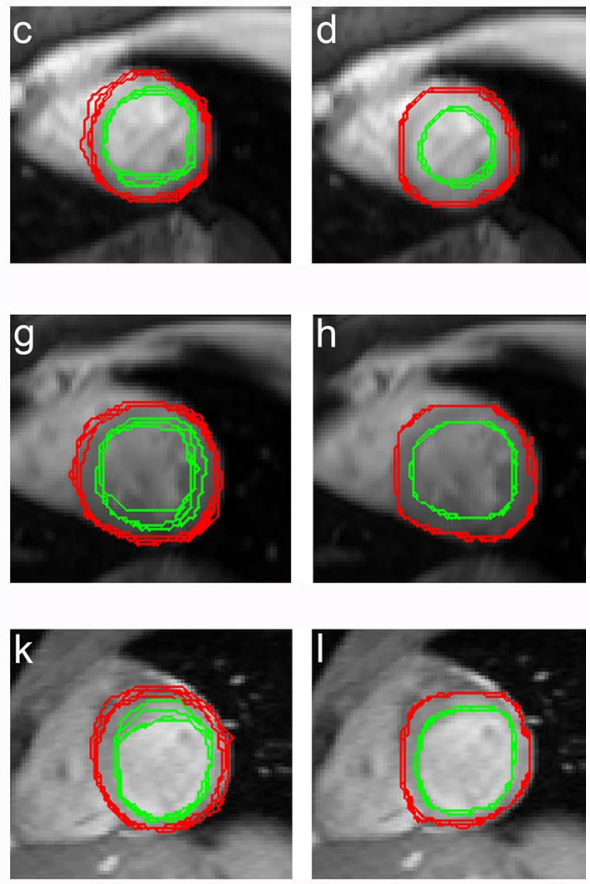

Manual

Semi-automatic of experience in cardiovascular imaging set the window level and width of all images so that they were equal for all observers. The semi-automatic myocardial segmentation was then conducted on the two sets of previously generated composite images. The time spent for the generation of the composite images by the two methods, and for drawing the manual and semi-automatic myocardial contours, was recorded automatically by the software. RBP and JMvS had profound knowledge of short-axis myocardial anatomy and are further referred to as "non-radiologists". Myocardial contour agreement within and between observers using manual drawing and semi-automatic segmentation were assessed by the dice similarity coefficient (DSC):

$\mathrm{DSC}=(A, B)=\frac{2(A \cap B)}{(A+B)}$

where $A$ and $B$ represent the contour regions and $\cap$ and + represent the intersection and addition between regions, respectively, with a minimum DSC value of 0 which indicates no contour agreement and a maximum value of 1 in case of total overlap [30].

\section{Statistical analysis}

Agreements on myocardial area and contour similarity (DSC) were assessed and presented as median \pm median absolute deviation (MAD). A paired Wilcoxon test was used to compare the DSC agreement of manual drawing and semi-automatic segmentation conducted on the composite images generated by the two methods. The Bland-Altman analysis [38] of segmental T2* reproducibility between and within observers as assessed by manual and semi-automatic segmentation is presented by mean difference \pm limit of agreement (LoA). The LoA is defined as $1.96 \times$ standard deviation (SD) of the difference. The identification of a segment with the minimum $\mathrm{T} 2 *$ value per slice was based on the result of semi-automatic segmentation of the composite images generated by method 2 (that turned out to have highest performance in contour reproducibility). Based on this quantification, two groups with and without local iron deposition were identified (T2* value $\leq 20$ and $>20 \mathrm{~ms}$, respectively) $[39,40]$.

Intraobserver and interobserver reproducibility of manual and semi-automatic segmentation using the two methods was assessed in the following groups; all observers, radiologist, non-radiologist, and in the pathology groups of with and without local iron deposition. Segmental T2* analysis between and within observers was performed in the same way as described for the myocardial contours. Variability was presented by the $\mathrm{CoV}$, expressed as a percentage. Reliability was presented as the two-way random intra-class correlation coefficient (ICC), evaluating absolute agreement. The Friedman test was used to assess the reproducibility of optimal TE image selection and CNR improvement of the composite image between observers. IBM SPSS Statistics software version 20 (IBM Corporation, Somers, NY, USA) was used to perform all statistical analyses, with $P<0.05$ being considered as statistically significant. 


\section{Results}

\section{CNR based on composite image generation}

In this study, two different methods of selecting the myocardial surrounding regions used in the generation of the contrast-optimized composite images were evaluated. Manual input per patient (three short-axis slices) to generate the composite image by method 1 was small compared with method 2 (mean \pm SD of $41.19 \pm 4.80 \mathrm{~s}$ vs. $96.12 \pm 13.98 \mathrm{~s}$, $P<0.01)$. Thus, two or three images from the MGE series were generally selected and weighted in a ratio of two to one (two images) or equally (three images) [30] to produce the composite images (43.52 and 55.50\% in method 1, and 50.49 and $49.51 \%$ in method 2 , respectively). In method 1 , the longest TE image (8th TE of 18.20 or $18.86 \mathrm{~ms}$, scanned by the Avanto or Aera scanner, respectively) was commonly selected as a representation of maximum CNR between LV myocardium and both LVBP (65.69\%), and RVBP (32.60\%), while the image of the second TE (4.82 or $5 \mathrm{~ms}$ by the Avanto or Aera scanner, respectively) was chosen for maximum CNR between LV myocardium and lung (47.79\%). The same pattern was observed in the image selection in method 2, where the maximum CNR between the LV myocardium and both LVBP and RVBP were observed at the longest TE (72.79 and $54.17 \%$, respectively), and between the LV myocardium and lung at the shortest TE ( 2.59 or $2.69 \mathrm{~ms}$ by the Avanto or Aera scanner, respectively; 83.09\%).

In method 2, reproducibility of selecting the same MGE images for maximum CNR between the LV myocardium and its surroundings was higher than in method 1 , as evidenced by higher ICC when using method 2 compared to method 1 ( 0.981 vs. 0.897 between the LV myocardium and LVBP, 0.951 vs. 0.877 between the LV myocardium and RVBP, and 0.947 vs. 0.862 between the LV myocardium and lung). Only in method 1 was a significant difference was found between observers in MGE image selection for maximum CNR between the LV myocardium and RVBP $(P<0.05)$. Using method 2 for generation of the composite image, CNR gains (relative to the highest CNR of any original MGE image) were consistently higher as compared to method 1 between the LV myocardium and LVBP [mean $80.87 \%, 95 \%$ confidence interval (CI) of $74.89-86.85 \%$ vs. mean $45.32 \%, 95 \%$ CI of 39.23-51.42\%], between the LV myocardium and RVBP (mean $94.71 \%, 95 \%$ CI of 86.79 $102.63 \%$ vs. mean $78.36 \%, 95 \%$ CI of $69.69-87.04 \%$ ), and between the LV myocardium and lung (mean $69.19 \%$, $95 \%$ CI of $62.94-75.44 \%$ vs. mean $91.92 \%, 95 \%$ CI of 84.32 $99.51 \%$ ). On average, the composite images improved contrast between the LV myocardium and its surroundings by $81.48 \%$ (95\% CI of 77.55-85.43\%) using method 2 and by $71.87 \%$ (95\% CI of $67.40-76.34 \%$ ) using method 1 above the maximum CNR at any single TE.

\section{Reproducibility of myocardial contours}

The observers produced the contours by manual drawing and semi-automatic segmentation (three short-axis slices per patient) in, on average, $73.82 \pm 13.70 \mathrm{~s}$ and $14.26 \pm 0.74 \mathrm{~s}$, respectively $(P<0.001)$. Adding up the composite image generation time to that of the contour revealed that the semi-automatic segmentation method took less time than manual drawing by both method 1 (mean \pm SD of $55.58 \pm 4.90$ vs. $107.13 \pm 12.01 \mathrm{~s}$, $P<0.001$ ), and method 2 (mean \pm SD of $110.25 \pm 13.56$ vs. $177.83 \pm 27.93 \mathrm{~s}, P<0.001)$.

Intraobserver reproducibility analysis of 51 short-axis slices yields a total of $(51 \times 4=204)$ DSCs contours while, for interobserver reproducibility, the total DSCs becomes $(51 \times 24=1224)$ DCSs. For all observers, intraobserver reproducibility of myocardial contours on contrast-optimized composite images generated by the two methods improved when using the semi-automatic segmentation compared to manual drawing (DSC of $0.86 \pm 0.05$ vs. $0.83 \pm 0.04, P<0.001$ by method 1 and DSC of $0.86 \pm 0.05$ vs. $0.81 \pm 0.03, P<0.001$ by method 2 ). The same trend of DSCs improvement by using the semiautomatic segmentation on method 1 (Table 1) and method 2 (Table 2) was also found within observers of different experience level and when the data were differentiated into short-axis slices with segmental T2* $\leq 20$ and $>20 \mathrm{~ms}$. The only exception is that DSC improvement within non-radiologists lacked significance on method $1(P>0.05)$. Between all observers, interobserver reproducibility was improved by using the semi-automatic segmentation compared to manual drawing when using both method $1(P<0.001)$ and method $2(P<0.001)$ and likewise in all subgroups with better DSCs generally acquired when using the semi-automatic segmentation rather than manual drawing (Tables 1 and 2).

In the first columns of Tables 1 and 2, LV myocardial areas measured by manual drawing and semi-automatic segmentation are presented in percentages of the average area per observer and pathology group, with the collective areas in each group were also expressed in $\mathrm{mm}^{2}$ between brackets. The radiologists tended to get smaller areas $(83-87 \%)$ by the manual drawing with the two methods than the non-radiologists (111-117\%). This variation is reduced by the semi-automatic segmentation with the two methods as performed by the radiologists (99-100\%) and the non-radiologists (99-101\%). Measurement bias of the LV myocardial area between all observers was also reduced by using the semi-automatic segmentation as compared to manual drawing (Fig. 6), both by using method 1 (mean difference $\pm \mathrm{LoA}$; CoV of $-20.57 \pm 562.43 \mathrm{~mm}^{2}$; $21.60 \%$ vs. $-332.44 \pm 617.02 \mathrm{~mm}^{2} ; 22.88 \%$ ), and method 2 (mean difference $\pm \mathrm{LoA}$; $\mathrm{CoV}$ of $-18.53 \pm 489.22 \mathrm{~mm}^{2}$; 


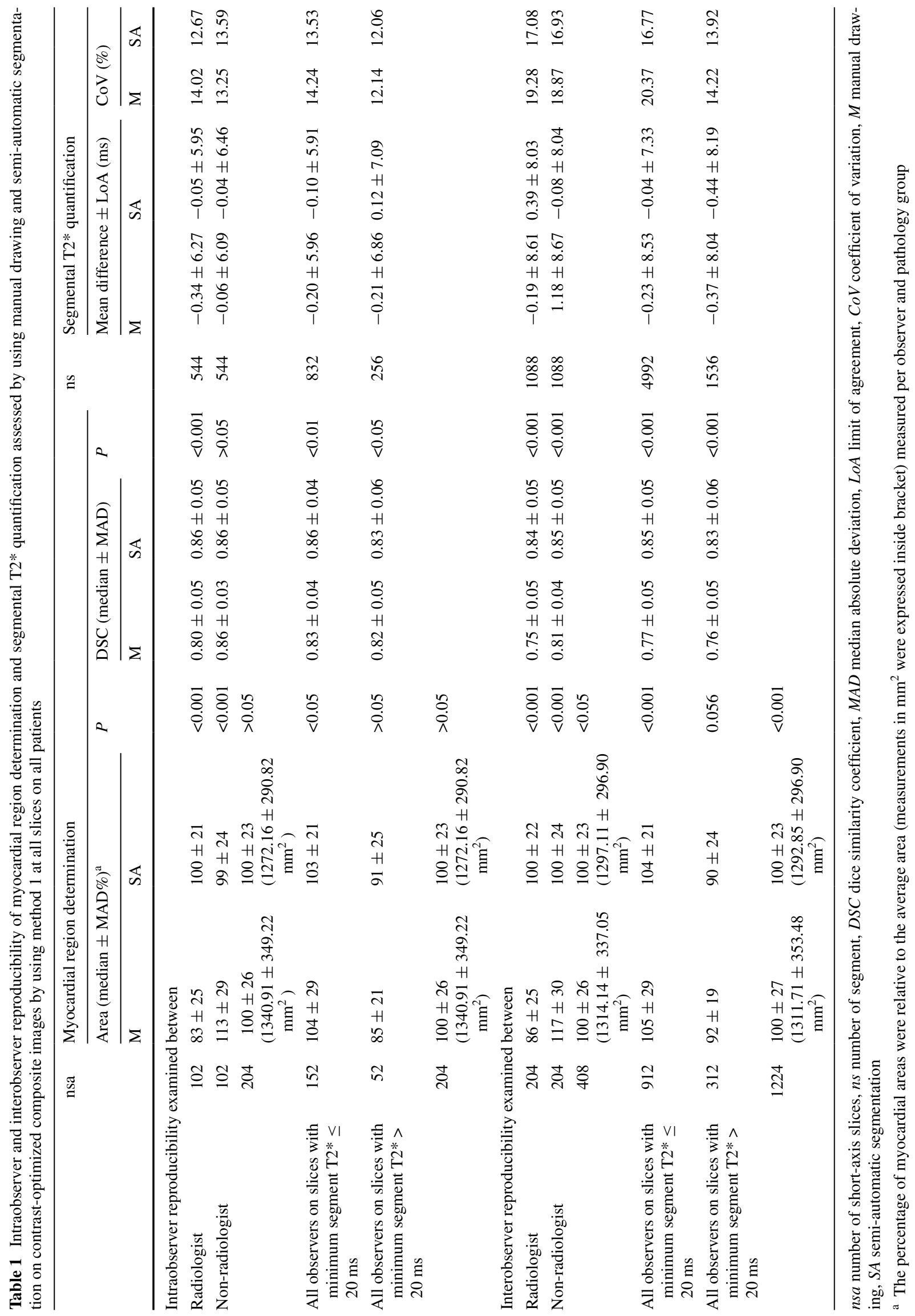




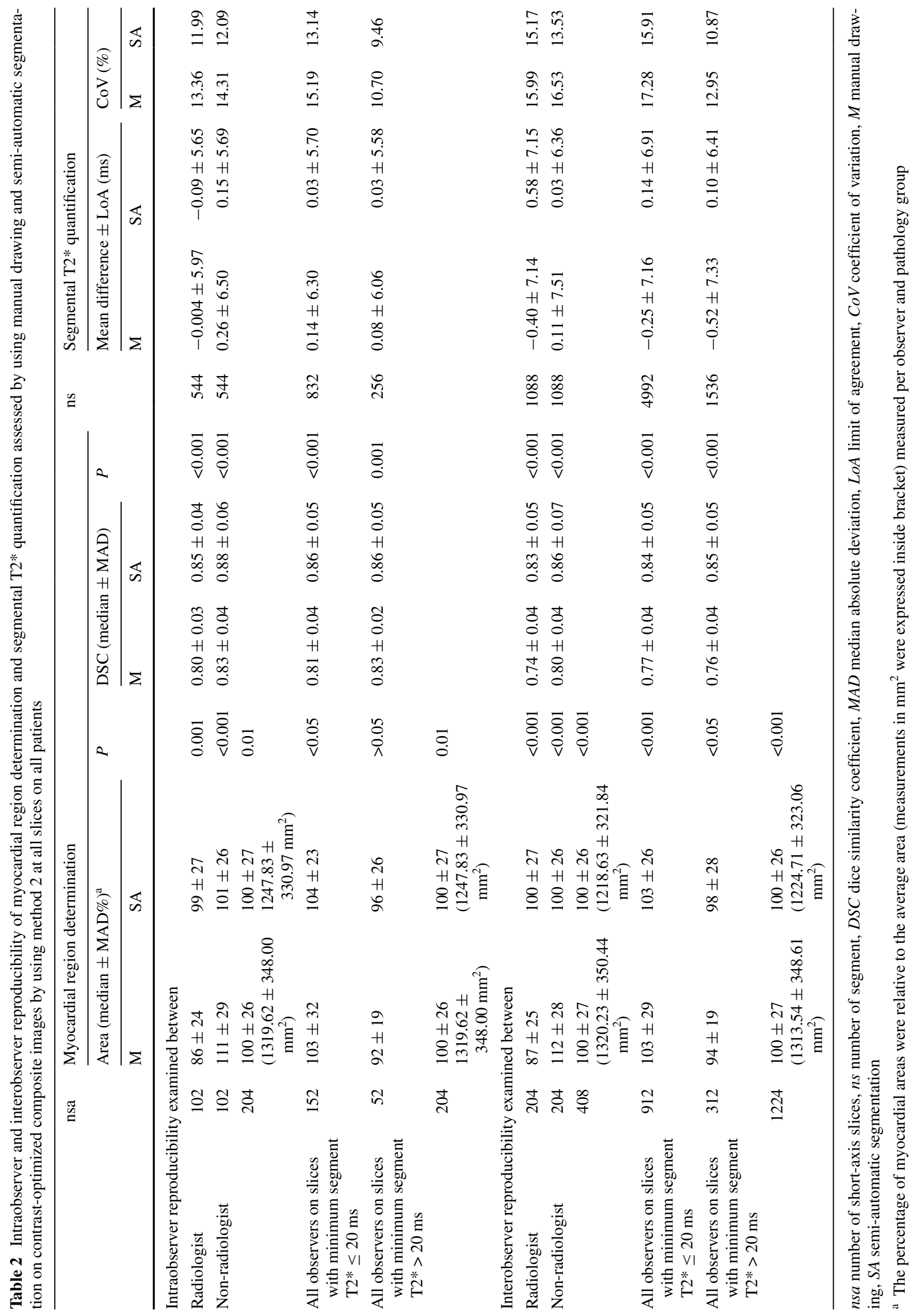


Fig. 6 Bland-Altman plots between all observers assessing myocardial area on contrastoptimized composite images generated by method 1 on 17 patients at apical, mid-ventricular and basal locations (a total of 1224 short-axis slices) using manual drawing (a) and semiautomatic segmentation (b) and by method 2 using manual drawing (c) and semi-automatic segmentation (d) a

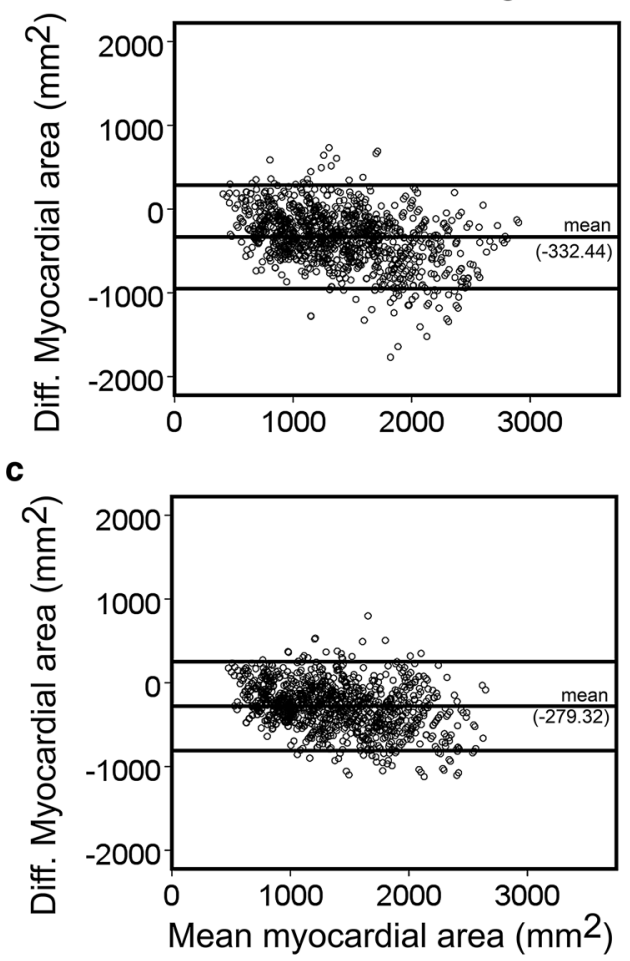

b Semi-automatic segmentation

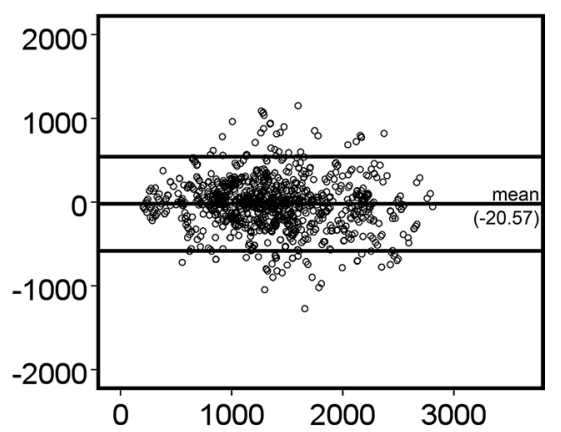

d

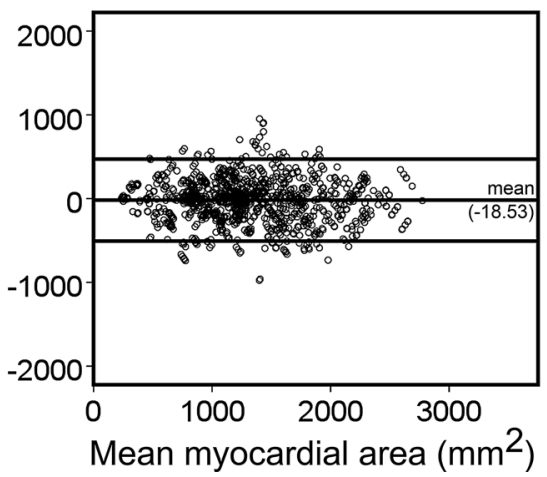

$19.30 \%$ vs. $-279.32 \pm 530.24 \mathrm{~mm}^{2} ; 19.88 \%$ ). A similar trend between all observers was found for slices with (mean difference $\pm \mathrm{LoA}$; $\mathrm{CoV}$ of $-18.45 \pm 521.04 \mathrm{~mm}^{2}$; $20.05 \%$ vs. $-285.92 \pm 553.45 \mathrm{~mm}^{2} ; 20.12 \%$ ) and without the presence of local iron loading (mean difference $\pm \mathrm{LoA}$; $\mathrm{CoV}$ of $-18.75 \pm 382.00 \mathrm{~mm}^{2} ; 16.26 \%$ vs. $\left.-260.02 \pm 454.37 \mathrm{~mm}^{2} ; 18.74 \%\right)$.

\section{Reproducibility of segmental myocardial T2*}

Fifty-one short-axis slices of 17 patients at apical, mid-ventricular, and basal locations generated a dataset containing 272 myocardial segments according to the AHA 16-segment model. Evaluation of minimum segmental T2* values $\leq 20$ ms revealed 38 slices with local iron deposition on 10 apical (eight at anterior, one at septal, seven at inferior, and five at lateral segments), 13 mid-ventricular (eight at anterior, two at anteroseptal, four at inferoseptal, six at inferior, eight at inferolateral, and four at anterolateral segments), and 15 basal locations (nine at anterior, five at anteroseptal, two at inferoseptal, four at inferior, eight at inferolateral, and five at anterolateral segments). Bland-Altman analysis of segmental T2* quantification using the manual drawing and semi-automatic segmentation is shown in Tables 1 and 2 for the assessment by methods 1 and 2 .

In general, compared to method 1 , segmental $\mathrm{T} 2 *$ quantification on the contrast-optimized composite image generated by method 2 shows lower LoA and CoV in intraobserver and interobserver groups assessed both by the semi-automatic segmentation and manual drawing. When intraobserver and interobserver variation is considered for all observers, lower LoA and $\mathrm{CoV}$ of $\mathrm{T} 2 *$ are obtained by the semi-automatic segmentation than by manual drawing generated both by method 1 and method 2. Consistent reductions of $\mathrm{LoA}$ and $\mathrm{CoV}$ of $\mathrm{T}_{2} *$ were also found in intraobserver and interobserver subgroups by using the semi-automatic segmentation compared to manual drawing on method 2 , while in method 1 , inconsistent reductions were found in intraobserver variance between nonradiologists. Focusing on intraobserver and interobserver variation of $\mathrm{T} 2 *$ quantification in the global $\mathrm{LV}$ heart as compared with the mid-ventricular septum (Table 3) evaluation shows that the semi-automatic segmentation produces lower observer variability of $\mathrm{T} 2 *$ quantification compared to manual drawing in both global LV and mid-ventricular septal with the least spread when using method 2 .

In the analysis of myocardial $\mathrm{T} 2 * \leq 20 \mathrm{~ms}$ as an indication of iron deposition, consistent reductions of LoA and $\mathrm{CoV}$ of segmental $\mathrm{T} 2 *$ within and between all observers were found on the semi-automatic segmentation compared to manual drawing assessed on both methods with the same trends to those observed for segmental T2* $>20 \mathrm{~ms}$. The Bland-Altman plots between all observers quantifying segmental T2* $\leq 20$ and $>20 \mathrm{~ms}$ by using the semi-automatic segmentation and manual drawing on method 2 are shown in Fig. 7. 
Table 3 Global and midventricular myocardial $\mathrm{T} 2 *$ coefficient of variance $(\mathrm{CoV})$ of all patients assessed within and between all observers using manual drawing and semiautomatic segmentation on contrast-optimized composite images generated by two methods

\begin{tabular}{|c|c|c|c|c|c|c|}
\hline \multirow[t]{3}{*}{ Segmental analysis } & \multirow[t]{3}{*}{ ns } & \multicolumn{2}{|l|}{ Intraobserver } & \multirow[t]{3}{*}{ ns } & \multicolumn{2}{|l|}{ Interobserver } \\
\hline & & Manual & Semi-automatic & & Manual & Semi-automatic \\
\hline & & $\mathrm{T} 2 * \operatorname{CoV}(\%)$ & $\mathrm{T} 2 * \operatorname{CoV}(\%)$ & & $\mathrm{T} 2 * \operatorname{CoV}(\%)$ & $\mathrm{T} 2 * \operatorname{CoV}(\%)$ \\
\hline \multicolumn{7}{|l|}{ Method 1} \\
\hline Global myocardium & 1088 & 13.64 & 13.14 & 6528 & 18.57 & 15.97 \\
\hline $\begin{array}{l}\text { Mid-ventricular septal } \\
\text { Method } 2\end{array}$ & 136 & 9.73 & 7.83 & 816 & 14.92 & 11.20 \\
\hline Global myocardium & 1088 & 13.86 & 12.05 & 6528 & 16.01 & 14.43 \\
\hline Mid-ventricular septal & 136 & 10.87 & 9.73 & 816 & 11.89 & 9.68 \\
\hline
\end{tabular}

$n s$ number of segment, $C o V$ coefficient of variation
Fig. 7 Bland-Altman plots between all observers assessing myocardial $\mathrm{T} 2 *$ on short-axis slices by manual drawing (a) and semi-automatic segmentation (b) on contrast-optimized composite image generated by method 2 in the presence of at least a myocardial segment with minimum $\mathrm{T} 2 * \leq 20 \mathrm{~ms}$ (4992 segments) and by manual drawing (c) and semi-automatic segmentation (d) in the absence of any segment with minimum $\mathrm{T} 2 * \leq 20 \mathrm{~ms}$ (1536 segments) a

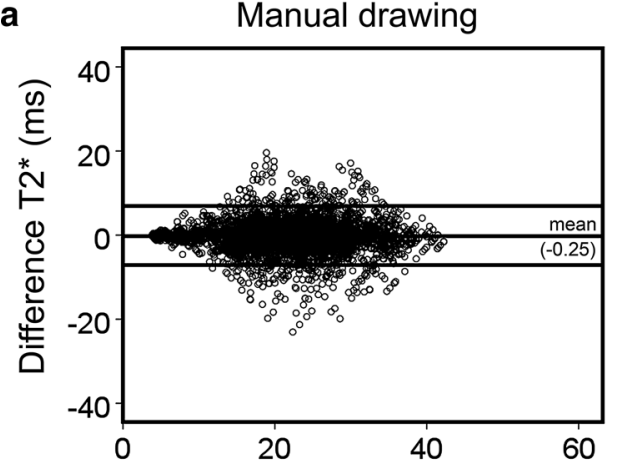

C

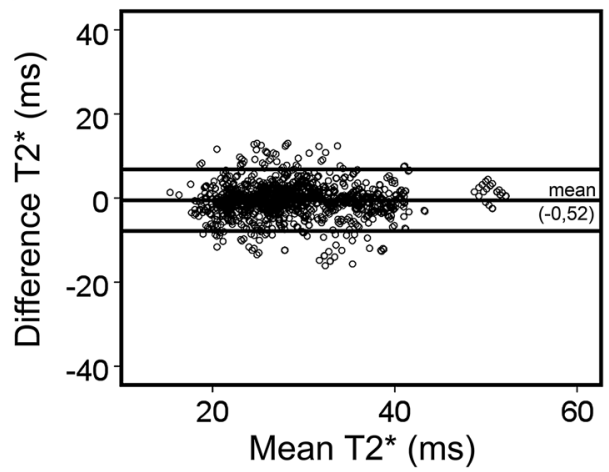

b Semi-automatic segmentation

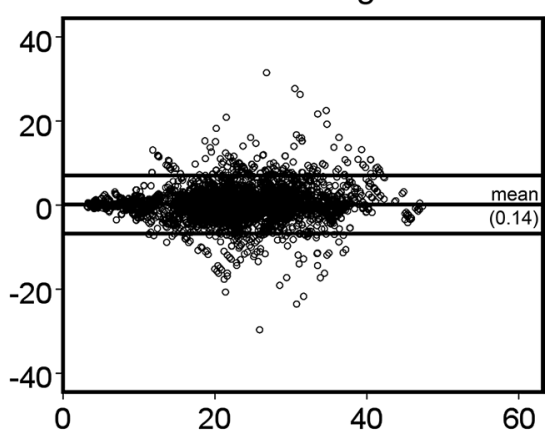

d

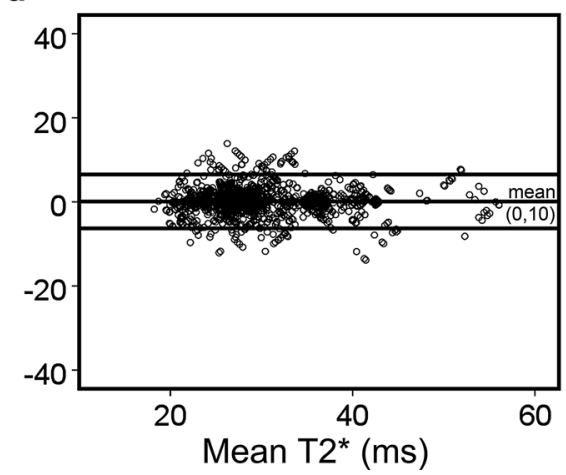

\section{Discussion}

This study shows that semi-automatic myocardial segmentation improves reproducibility within and between observers in defining myocardial area as compared to manual drawing with an almost five times faster post-processing time without significant elimination of pixels at the myocardial borders. This result is important to achieve reproducible and reliable myocardial $\mathrm{T} 2 *$ quantification, especially for thalassemia and suspected cardiomyopathy patients where evidence shows that early stage of iron deposition starts from the epicard [11, 13, 14, 41, 42]. Contour reproducibility is beneficial in the clinical evaluation of patients with iron loading indication, where our novel procedure reduces the variability in segmental myocardial $\mathrm{T} 2 *$ quantification (Table 2). The $\mathrm{T} 2 *$ approach presented in this study is not limited to mid-ventricular septum analysis, but also successfully applied for global analysis identifying local heterogeneity of iron deposition on different locations of the LV heart. This finding indicates that the semiautomatic segmentation can replace the standard manual approach (Table 3). Even though MGE acquisition was done on two different scanners in this study, other studies have confirmed the reproducibility of myocardial $\mathrm{T} 2 *$ data from different centers operating different scanners [43, 44]. The LV myocardial contour improvement in this study is 
achieved by using the contrast-optimized composite images generated by method 2, which, on average, improves the CNR between myocardium and its surrounding regions by $81.48 \%$.

The use of contrast-optimized composite images in improving the CNR between myocardium and its surroundings has been shown previously [30]. In that study, manual location and drawing of ROIs in the surroundings of the LV myocardium served to select those three images from the MGE series to be merged into composite images based on maximum CNRs. It was the intention in this study to replace that rather subjective approach with a more systematic approach so that a more reproducible TE image combination can be produced.

In this study, two methods for generating the combination of three TE images were, therefore, assessed and evaluated. Although method 1 was even faster $(P<0.001)$, method 2, with a more objective approach, produced higher reliability (higher ICC) with a higher percentage rate of the same image selection. This improved the CNR gain between the LV myocardium and its surroundings on the composite images compared to method 1 ( 81.48 vs. $71.87 \%)$. The drawback of using method 1 in producing a reliable MGE image selection is in the use of an anterior RV-LV insertion point as reference to create the six segments and the fixed positions of RVBP and lung ROIs at the edge of the segments, rendering the procedure more observer-dependent and eliminating the flexibility to adapt the lung (Fig. 2c) and RV (Fig. 2b) sizes. This drawback influences the generated composite image due to remaining variation in the selection of images providing optimum contrast between the LV myocardium and RVBP and between the LV myocardium and lung. Our results show that the generation of a composite image is made by mostly combining images at the first and the last TE, similar to previous study [30]. However, higher repeatability was achieved in this study when comparing the percentages of TE image selection (proven also by high ICCs) implying superiority of our approach (method 2) in generating contrast-optimized composite images compared with the previous study.

Unlike the black blood MGE series [26], blood signal artifacts and partial volume effects in bright blood MGE series create signal inhomogeneity in LVBP, making the segmentation of LVBP contours challenging. In this study, the subtraction of the shortest TE image from that with optimum TE between the LV myocardium and LVBP was proven as an effective technique to reduce inhomogeneity by creating a low signal intensity image. Due to the presence of artefacts in the bright blood mode, the homogeneous signal at the center of the LVBP is not circularly shaped and, therefore, the circular Hough transformation proposed by Zheng et al. [26] cannot be implemented as a start for LVBP area detection. Therefore, we combined $k$-means clustering together with VFC active contours to encounter the LVBP border detection problem on all short-axis images acquired not only on mid-ventricular but also on apical and basal locations. Others reported the use of watershed segmentation after imaging morphological processes on the bright blood mode to acquire a mask image for the LVBP boundary detection by Geodesic active contours and Level set [27]. But the combination of these methods still introduces a high false detection of LVBP centroid leading to false endocardial contour determination. As proposed by Lynch et al. [32], the first step in contouring is the key to success for a robust active contour segmentation. The use of a manually drawn LV myocardial wall contour as the initial active contour in this study did influence the robustness of LVBP determination generated by different observers, especially in apical locations, as seen in Fig. 5b, d. Nevertheless, variability in contours was lower than that produced with manual contour determination (Fig. 5).

We introduced a new technique to determine the epicardial border of LV myocardium by using mean signal intensity information of the LV myocardial wall located on several radial pixel's segments and layers. CoV assessment and $k$-means clustering were then applied in the combination of these segments and layers to determine the epicardial border. The $\mathrm{CoV}$ was proven to be effective in determining the lateral border, while the $k$-means clustering was able to determine the anterior, septal, and posterior borders. By further automated correction procedures, low-contrast problems interfering with the depiction of the epicardial border at septal, anterior, and posterior locations could be solved. Prior knowledge of the LV myocardial wall was used in this technique to ensure the effectiveness of the clustering method in determining the epicardial border location.

The semi-automatic myocardial segmentation in this study reduces the post-processing time to several orders of magnitude shorter than that reported elsewhere [27] and yields higher intraobserver and interobserver reproducibility as compared to manual drawing, leading to lower variability in myocardial $\mathrm{T} 2 *$ quantification regardless of the presence or absence of iron loading pathology $(\mathrm{T} 2 * \leq 20$ and $>20 \mathrm{~ms}$ ). The results show that the semi-automatic segmentation method produces roughly the same area of myocardium compared to manual drawing (Tables 1 and 2, area of LV myocardial region determination) with the advantage of lower variability in area measurement (lower SD in general) and substantial reduction of measurement bias between observers (Fig. 6). Compared to other study [30], here higher observer variability in $\mathrm{T} 2 *$ quantification is influenced by subjective input, reflecting the observer's background in defining the myocardial area and is reduced by using the semi-automatic segmentation (higher DSC with $P<0.001$ ). Another reason is the heterogeneity of myocardial $\mathrm{T} 2 *$ in patients with borderline iron deposition in this study. 
Consistence of areas regardless of the observer's background make the segmentation method in this study a reliable LV myocardial area detection method for $\mathrm{T} 2 *$ quantification in the presence and absence of iron deposition (Figs. 5, 6). Our results are consistent with another study showing that radiologists tend to create tighter myocardial area definition than automatic detection [27]. Combining LV myocardial area analysis with contour shape analysis (DSC) in this study revealed that the semi-automatic segmentation produces more reproducible LV myocardial contours as compared to manual drawing while maintaining myocardial pixels near the endocardial and epicardial borders. This indicates that the possible loss of biologic information contained in low-T2* pixels at the periphery is negligible with the semi-automated segmentation, especially method 2. In the end, the global and mid-ventricular septal myocardial T2* quantification (Table 3 ) and the semi-automatic segmentation lowered variability between observers.

This study has a limitation in that the validation of the semi-automatic segmentation was done on a small sample of patient data in accordance with previous methodological studies $[19,27]$. The next step will be to validate the new method in a larger patient group in clinical practice. The monoexponential fitting model with constant offset used in this study yields only a small systematic bias for patients with borderline iron loading [4] compared to other fitting methods recently used $[9,16,45]$. The use of a combined truncation method with image corrections $[9,16,45]$ might be considered in future work. Here, local susceptibility artifacts might have influenced $\mathrm{T} 2 *$ heterogeneity even though its presence, after correction, is known to not significantly affect global heart T2* heterogeneity $[18,46]$. Another suggestion made in the literature is the use of susceptibility correction [15-18]. Maximum myocardial layer expansion in the semi-automatic segmentation was set at $17 \mathrm{~mm}$ from the epicardial border, an optimization which in the application for hypertrophic cardiomyopathy patients can only be applied for mild LV wall thickening identification [47]. Prior pathology information of images with more severe hypertrophic condition could be added in the segmentation procedure to customize the myocardial layer expansion on specific myocardial thickening regions, i.e. $>30 \mathrm{~mm}$ [48]. Even though method 2 has shown an improvement in selecting MGE images for the composite combination, the required manual drawing of RVBP and lung areas still is time-consuming. Manual definition of the myocardial wall to start the semi-automatic segmentation process causes some variation in the results within and between observers and increases the time expenditure for contour generation. Here, the developed semi-automatic segmentation method was only applied on a bright blood MGE image series showing poor contrast differences between the myocardium and its surroundings. Successful implementation of an entirely automatic approach to select the region by using boundary-based or region-based methods $[19,20]$ to shorten the process, and further improve the reproducibility of bright blood MRI might be a next step. In the meantime, it will be of interest to further validate our segmentation method in a black blood MGE series in comparison with alternatives provided by others.

\section{Conclusion}

In conclusion, the proposed semi-automatic myocardial segmentation as assessed on contrast-optimized composite images provides comparable and reproducible $\mathrm{T} 2 *$ quantification faster than manual drawing, and can be applied in clinical practice for global heart and mid-ventricular septum analysis. The effectiveness of the segmentation is influenced by the contrast difference between the myocardium and its surrounding tissues where, in this study, a CNR gain of, on average, $81.48 \%$ was achieved. In iron loading assessment, the proposed segmentation method leads to more consistent and less user-dependent myocardial detection areas resulting in better reproducibility of $\mathrm{T} 2 *$ quantification.

Acknowledgements Pandji Triadyaksa received funding support from DGHE scholarship of the Ministry of Research, Technology and Higher Education of Indonesia.

\section{Compliance with ethical standards}

Conflict of interest The authors declare that they have no conflicts of interest.

Ethical approval All procedures performed in studies involving human participants were in accordance with the ethical standards of the institutional and/or national research committee and with the 1964 Helsinki Declaration and later amendments or comparable ethical standards.

Informed consent For this type of study, formal consent is not required.

Open Access This article is distributed under the terms of the Creative Commons Attribution 4.0 International License (http://creativecommons.org/licenses/by/4.0/), which permits unrestricted use, distribution, and reproduction in any medium, provided you give appropriate credit to the original author(s) and the source, provide a link to the Creative Commons license, and indicate if changes were made.

\section{References}

1. Meloni A, Positano V, Ruffo GB, Spasiano A, D'Ascola DG, Peluso A, Keilberg P, Restaino G, Valeri G, Renne S, Midiri M, Pepe A (2015) Improvement of heart iron with preserved patterns of iron store by CMR-guided chelation therapy. Eur Heart J Cardiovasc Imaging 16:325-334 
2. Gulati V, Harikrishnan P, Palaniswamy C, Aronow WS, Jain D, Frishman WH (2014) Cardiac involvement in hemochromatosis. Cardiol Rev 22:56-68

3. He T (2014) Cardiovascular magnetic resonance $\mathrm{T} 2 *$ for tissue iron assessment in the heart. Quant Imaging Med Surg 4:407-412

4. Meloni A, Rienhoff HY, Jones A, Pepe A, Lombardi M, Wood JC (2014) Cardiac R2* values are independent of the image analysis approach employed. Magn Reson Med 72:485-491

5. Meloni A, Puliyel M, Pepe A, Berdoukas V, Coates TD, Wood JC (2014) Cardiac iron overload in sickle-cell disease. Am J Hematol 89:678-683

6. Baksi AJ, Pennell DJ (2014) T2* imaging of the heart. Top Magn Reson Imaging 23:13-20

7. He T, Gatehouse PD, Kirk P, Tanner MA, Smith GC, Keegan J, Mohiaddin RH, Pennell DJ, Firmin DN (2007) Black-blood T2* technique for myocardial iron measurement in thalassemia. J Magn Reson Imaging 25:1205-1209

8. Meloni A, Restaino G, Borsellino Z, Caruso V, Spasiano A, Zuccarelli A, Valeri G, Toia P, Salvatori C, Positano V, Midiri M, Pepe A (2014) Different patterns of myocardial iron distribution by whole-heart $\mathrm{T} 2 *$ magnetic resonance as risk markers for heart complications in thalassemia major. Int J Cardiol 177:1012-1019

9. Kellman P, Xue H, Spottiswoode BS, Sandino CM, Hansen MS, Abdel-Gadir A, Treibel TA, Rosmini S, Mancini C, Bandettini WP, McGill L-A, Gatehouse P, Moon JC, Pennell DJ, Arai AE (2015) Free-breathing T2* mapping using respiratory motion corrected averaging. J Cardiovasc Magn Reson 17:3

10. Modell B, Khan M, Darlison M (2000) Survival in $\beta$-thalassaemia major in the UK: data from the UK Thalassaemia Register. Lancet 355:2051-2052

11. House MJ, Fleming AJ, de Jonge MD, Paterson D, Howard DL, Carpenter J-P, Pennell DJ, St Pierre TG (2014) Mapping iron in human heart tissue with synchrotron $\mathrm{x}$-ray fluorescence microscopy and cardiovascular magnetic resonance. J Cardiovasc Magn Reson 16:80

12. Olson LJ, Edwards WD, McCall JT, Ilstrup DM, Gersh BJ (1987) Cardiac iron deposition in idiopathic hemochromatosis: histologic and analytic assessment of 14 hearts from autopsy. J Am Coll Cardiol 10:1239-1243

13. Carpenter JP, He T, Kirk P, Roughton M, Anderson LJ, De Noronha SV, Sheppard MN, Porter JB, Walker JM, Wood JC, Galanello R, Forni G, Catani G, Matta G, Fucharoen S, Fleming A, House MJ, Black G, Firmin DN, St. Pierre TG, Pennell DJ (2011) On T2* magnetic resonance and cardiac iron. Circulation 123:1519-1528

14. Ghugre NR, Enriquez CM, Gonzalez I, Nelson MD, Coates TD, Wood JC (2006) MRI detects myocardial iron in the human heart. Magn Reson Med 56:681-686

15. Meloni A, Hezel F, Positano V, Keilberg P, Pepe A, Lombardi M, Niendorf T (2014) Detailing magnetic field strength dependence and segmental artifact distribution of myocardial effective transverse relaxation rate at $1.5,3.0$, and 7.0 T. Magn Reson Med 71:2224-2230

16. Sandino CM, Kellman P, Arai AE, Hansen MS, Xue H (2015) Myocardial T2* mapping: influence of noise on accuracy and precision. J Cardiovasc Magn Reson 17:7

17. Taylor BA, Loeffler RB, Song R, McCarville ME, Hankins JS, Hillenbrand CM (2013) Automated T(2) * measurements using supplementary field mapping to assess cardiac iron content. J Magn Reson Imaging 38:441-447

18. Positano V, Pepe A, Santarelli MF, Scattini B, De Marchi D, Ramazzotti A, Forni G, Borgna-Pignatti C, Lai ME, Midiri M, Maggio A, Lombardi M, Landini L (2007) Standardized T2* map of normal human heart in vivo to correct T2* segmental artefacts. NMR Biomed 20:578-590
19. Petitjean C, Dacher J-N (2011) A review of segmentation methods in short axis cardiac MR images. Med Image Anal 15:169-184

20. Wang L, Chitiboi T, Meine H, Günther M, Hahn HK (2016) Principles and methods for automatic and semi-automatic tissue segmentation in MRI data. Magn Reson Mater Phy 29:95-110

21. Codella NCF, Weinsaft JW, Cham MD, Janik M, Prince MR, Wang Y (2008) Left ventricle: automated segmentation by using myocardial effusion threshold reduction and intravoxel computation at MR imaging. Radiology 248:1004-1012

22. Lee HY, Codella NCF, Cham MD, Weinsaft JW, Wang Y (2010) Automatic left ventricle segmentation using iterative thresholding and an active contour model with adaptation on short-axis cardiac MRI. IEEE Trans Biomed Eng 57:905-913

23. Wang L, Pei M, Codella NCF, Kochar M, Weinsaft JW, Li J, Prince MR, Wang Y (2015) Left ventricle: fully automated segmentation based on spatiotemporal continuity and myocardium information in cine cardiac magnetic resonance imaging (LVFAST). Biomed Res Int. doi:10.1155/2015/367583

24. O'donnell T, Funka-Lea G, Tek H, Jolly MP, Rasch M, Rasch M, Setser R (2006) Comprehensive cardiovascular image analysis using MR and CT at Siemens corporate research. Int J Comput Vis 70:165-178

25. Lee HY, Codella N, Cham M, Prince M, Weinsaft J, Wang Y (2008) Left ventricle segmentation using graph searching on intensity and gradient and a priori knowledge (lvGIGA) for short-axis cardiac magnetic resonance imaging. J Magn Reson Imaging 28:1393-1401

26. Zheng Q, Feng Y, Wei X, Feng M, Chen W, Lu Z, Xu Y, Chen $\mathrm{H}, \mathrm{He} \mathrm{T}$ (2015) Automated interventricular septum segmentation for black-blood myocardial T2* measurement in thalassemia. $\mathrm{J}$ Magn Reson Imaging 41:1242-1250

27. Luo Y, Ko JK, Shi L, Guan Y, Li L, Qin J, Heng P-A, Chu WC, Wang D (2015) Myocardial iron loading assessment by automatic left ventricle segmentation with morphological operations and geodesic active contour on T2* images. Sci Rep 5:12438

28. Pennell DJ, Porter JB, Cappellini MD, Chan LL, El-Beshlawy A, Aydinok Y, Ibrahim H, Li CK, Viprakasit V, Elalfy MS, Kattamis A, Smith G, Habr D, Domokos G, Roubert B, Taher A (2012) Deferasirox for up to 3 years leads to continued improvement of myocardial $\mathrm{T} 2 *$ in patients with $\beta$-thalassemia major. Haematologica 97:842-848

29. Pennell DJ, Porter JB, Piga A, Lai YR, El-Beshlawy A, Elalfy M, Yesilipek A, Kilin Y, Habr D, Musallam KM, Shen J, Aydinok Y (2015) Sustained improvements in myocardial T2* over 2 years in severely iron-overloaded patients with beta thalassemia major treated with deferasirox or deferoxamine. Am J Hematol 90:91-96

30. Triadyaksa P, Handayani A, Dijkstra H, Aryanto KYE, Pelgrim GJ, Xie X, Willems TP, Prakken NHJ, Oudkerk M, Sijens PE (2016) Contrast-optimized composite image derived from multigradient echo cardiac magnetic resonance imaging improves reproducibility of myocardial contours and $\mathrm{T} 2 *$ measurement. Magn Reson Mater Phy 29:17-27

31. Comaniciu D, Meer P (2002) Mean shift: a robust approach toward feature space analysis. IEEE Trans Pattern Anal Mach Intell 24:603-619

32. Lynch M, Ghita O, Whelan PF (2006) Automatic segmentation of the left ventricle cavity and myocardium in MRI data. Comput Biol Med 36:389-407

33. Acton ST (2008) Automatic active model initialization via poisson inverse gradient. IEEE Trans Image Process 17:1406-1420

34. Li B, Acton ST (2007) Active contour external force using vector field convolution for image segmentation. IEEE Trans Image Process 16:2096-2106

35. Kawel N, Turkbey EB, Carr JJ, Eng J, Gomes AS, Hundley WG, Johnson C, Masri SC, Prince MR, van der Geest RJ, Lima JAC, 
Bluemke DA (2012) Normal left ventricular myocardial thickness for middle-aged and older subjects with steady-state free precession cardiac magnetic resonance: the multi-ethnic study of atherosclerosis. Circ Cardiovasc Imaging 5:500-508

36. Saiviroonporn P, Viprakasit V, Boonyasirinant T, Khuhapinant A, Wood JC, Krittayaphong R (2011) Comparison of the region-based and pixel-wise methods for cardiac $\mathrm{T} 2 *$ analysis in 50 transfusion-dependent Thai thalassemia patients. J Comput Assist Tomogr 35:375-381

37. Ferguson MR, Otto RK, Bender MA, Kolokythas O, Friedman SD (2013) Liver and heart MR relaxometry in iron loading: reproducibility of three methods. J Magn Reson Imaging 38:987-990

38. Bland JM, Altman DG (1986) Statistical methods for assessing agreement between two methods of clinical measurement. Lancet $1: 307-310$

39. Wood JC (2007) Magnetic resonance imaging measurement of iron overload. Curr Opin Hematol 14:183-190

40. Carpenter J-P, Roughton M, Pennell DJ (2013) International survey of $\mathrm{T} 2 *$ cardiovascular magnetic resonance in $\beta$-thalassemia major. Haematologica 98:1368-1374

41. Buja LM, Roberts WC (1971) Iron in the heart. Etiology and clinical significance. Am J Med 51:209-221

42. Pepe A, Lombardi M, Positano V, Cracolici E, Capra M, Malizia R, Prossomariti L, De Marchi D, Midiri M, Maggio A (2006) Evaluation of the efficacy of oral deferiprone in beta-thalassemia major by multislice multiecho T2*. Eur J Haematol 76:183-192
43. Kirk P, He T, Anderson LJ, Roughton M, Tanner MA, Lam WWM, Au WY, Chu WCW, Chan G, Galanello R, Matta G, Fogel M, Cohen AR, Tan RS, Chen K, Ng I, Lai A, Fucharoen S, Laothamata J, Chuncharunee S, Jongjirasiri S, Firmin DN, Smith GC, Pennell DJ (2010) International reproducibility of single breathhold T2* MR for cardiac and liver iron assessment among five thalassemia centers. J Magn Reson Imaging 32:315-319

44. He T, Zhang J, Carpenter J-P, Feng Y, Smith GC, Pennell DJ, Firmin DN (2013) Automated truncation method for myocardial T2* measurement in thalassemia. J Magn Reson Imaging 37:479-483

45. Positano V, Meloni A, Santarelli MF, Gerardi C, Bitti PP, Cirotto C, De Marchi D, Salvatori C, Landini L, Pepe A (2015) Fast generation of $\mathrm{T} 2 *$ maps in the entire range of clinical interest: application to thalassemia major patients. Comput Biol Med 56:200-210

46. Meloni A, Pepe A, Positano V, Favilli B, Maggio A, Capra M, Lo Pinto C, Gerardi C, Santarelli MF, Midiri M, Landini L, Lombardi M (2009) Influence of myocardial fibrosis and blood oxygenation on heart $\mathrm{T} 2 *$ values in thalassemia patients. J Magn Reson Imaging 29:832-837

47. Olivotto I, Gistri R, Petrone P, Pedemonte E, Vargiu D, Cecchi F (2003) Maximum left ventricular thickness and risk of sudden death in patients with hypertrophic cardiomyopathy. J Am Coll Cardiol 41:315-321

48. Jiji RS, Kramer CM (2011) Cardiovascular magnetic resonance: applications in daily practice. Cardiol Rev 19:246-254 